\title{
An Investigation on Deformation Behaviors of Energy Absorbers for Passenger Coaches
}

\author{
Ramazan ÖZMEN ${ }^{1}$, Mustafa GÜNAY ${ }^{2}$ \\ ${ }^{1}$ Department of Mechatronics Engineering,KarabükUniversity \\ ${ }^{2}$ Department of Mechanical Engineering, Karabük University
}

\begin{tabular}{l} 
Article Info \\
\hline Article history: \\
Received Jun $1^{\text {th }}, 2017$ \\
Revised Aug $20^{\text {th }}, 2017$ \\
Accepted Oct $18^{\text {th }}, 2017$ \\
\hline
\end{tabular}

\section{Keyword:}

Energy absorbtion Finite element analysis HSLA steel Truncated tube Optimization

\begin{abstract}
Thin-walled structures is used commonly as energy absorbers at the front and back of the coaches. These parts should be designed to minimize the damage to the vehicle and prevent the passengers from fatality and/or injury by absorbing the collision energy in railway transportation. In this paper, deformation behaviors of tube like structures with truncated cone under the axial impact load were investigated by means of finite element analysis (FEA). The energy absorbers having tube like structures were modelled at the same weight and have three different wall thickness and taper angle. As a result of FEA, the performances of straight and truncated cone type energy absorbers were compared in terms of energy absorption capacities and an optimization study was done to determine the effects of thickness and taper angle on energy absorbing performances of the members. The analysis of variance in $95 \%$ confidence level was applied in order to determine the effects of design parameters on total efficiency (TE). Besides, optimum design parameters for TE were determined by using Taguchi optimization methodology. Thickness was found as the most significant parameter on total efficiency with $60.52 \%$ percentage contribution ratio according to ANOVA results.
\end{abstract}

\section{Corresponding Author:}

First Author,

Department of Mechatronics Engineering,

Karabük University,

BalıklarkayasıMevkii,78050, Karabük, Turkey.

Email: ramazanozmen@karabuk.edu.tr

\section{Introduction}

In railway transport, collision zones have been developed in front and rear of the car to damp the collision energy to prevent catastrophic events such as accidental death and injury. During the accident, the kinetic energy is consumed in a controlled manner by way of these collision zones to preserve the integrity of the passengers and the occupied areas [1][2].

In order to absorb impact energy, thin-walled tube-like components are generally used as energy absorbing members in vehicles such as cars, planes and trains etc. The static and dynamic axial impact behaviors of these members with different cross sections (circular, rectangular etc.) were examined by means of several experimental and theoretical studies and certain theoretical expressions were suggested according to the crosssectional properties [3].

Energy absorption capabilities and axial impact behaviors of thin-walled tubes depend on many factors such as material properties [4], member geometry [5], impact velocity [6], applied boundary conditions [7], and forming history [8]. These studies have revealed that the hardening characteristics and strain rate sensivity of the material are significant effects on the collision behavior of the energy absorber [4]. On the other hand, it 
was stated that the stress-strain relationship of the material has a key place in the numerical simulation of the collision events in which the large deformations occurs [9].

In some studies, trigger structures like holes and groves have been utilized to provide a controlled deformation and to increase energy absorption. One of the most key features of this trigger mechanism is to reduce the initial peak force generated during the impact [10]. And, aluminum foams and honeycomb structures were placed inside the energy absorber to increase the energy absorption capability of the member [11].

The primary energy absorber is one of the crucial structure in the passenger wagon that is designed for the collapse of impact energy. The current primary energy absorber is comprised of two tube like structures in the form of square cross section. Holes are drilled on the member to improve energy absorption capability and the two members are joined to each other using welded intermediate diaphragm like members [12].

In this study, the deformation behaviors of tube like structures in the form of straight and truncated cone members were investigated by means of finite element analysis method. The investigated members have same weight and length and different wall thicknesses.

At the end of the study, the axial deformation performances of straight and truncated cone members were compared. An optimization study was done to determine the effects of thickness and taper angle on energy absorbing performances of the members.

\section{Material and Method}

\subsection{Material model and design parameters}

High-strength low alloy (HSLA) or micro alloyed steels present a yield strength of 340-420 MPa and ultimate tensile strength of $410-510 \mathrm{MPa}$, while their uniform elongation ranges between 10 and $25 \%$. Consequently, HSLA steels generally require 25-30\% more power to form due to of their higher strength and toughness, for example compared to carbon steels [13]. Thus, the steels are commonly used in trucks, construction equipment, off-highway vehicles, mining equipment, and heavy-duty vehicles for constructing chassis components, buckets and grader blades [14]. In the light of this knowledge, the HSLA 350 steel was chosen as material in the axial collision analysis of the energy absorbers or members used in the passenger coach. The design parameters for energy absorbers was selected as thickness (T) and taper angle (Ta), but the weights of circular section absorbers were kept as constant. The following design parameters were used for the finite element analyses; thickness of 4, 6 and $8 \mathrm{~mm}$, taper angles of $0,1.5$ and 3 degrees. In order to achieve the same weight value, the cross section of the absorber is increased as the thickness is decreased, and the member cross section is decreased as the thickness is increased.

In the analysis, the mass and speed values of the impacting wall, which was modelled for the axial deformation of the structure in the form of the tube, were taken from the experiments applied to the existing energy absorber used in passenger coaches [15]. The method and material properties used during modelling are given below. In the axial collision analysis of the energy absorbers made of HSLA350 material, stress strain diagrams of the material were used depending on the strain rate. The stress-strain diagram of the HSLA350 high strength steel, depending on the deformation rate, is given in Figure 1.

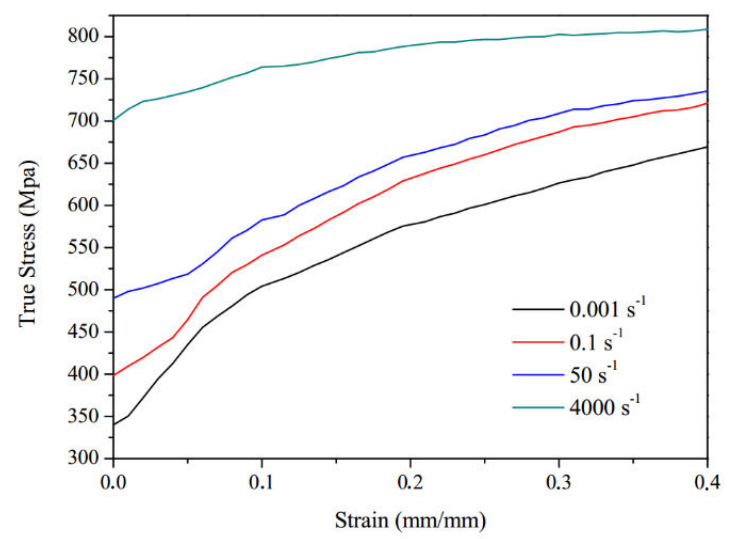

Figure 1. Stress-strain diagram of HSLA 350 steel [6] 
In LS-DYNA, these stress-strain values were defined using the Type-24 material model. This material model is defined as a piecewise linear isotropic elastic-plastic material model. In Type-24 material model, the effect of strain rate is achieved by Cowper-Symonds equation. In this equation, the yield stress is multiplied by the factor given below [16]. In the equation (1), $\sigma_{d}, \sigma_{s}, \dot{\varepsilon}, \mathrm{D}$ and $\mathrm{p}$ are dynamic yield stress, yield stress, deformation rate and Cowper-Symonds material constants, respectively.

$$
\sigma_{d}=\sigma_{y}[\dot{\varepsilon} / D]^{1 / p}
$$

\subsection{Finite Element Model}

In this study, the deformation behaviors of thin-walled tubes under axial load were examined by the software of LS-DYNA. When describing the boundary conditions in the finite element model, existing installation conditions were considered. Under existing conditions, one end of one energy absorbing member free and the other is welded to the frame of the CEM (Crash Energy Management) system (Fig. 1). For this reason, all the degrees of freedom of the base nodes of the modelled member were restricted and no boundary conditions were applied to the ceiling nodes. The impacting body was modeled with a rigid plate with a mass of $2500 \mathrm{~kg}$ and a velocity of $21 \mathrm{~m} / \mathrm{s}$. The finite element model of the energy absorber used in the analyses is given in Fig. 2 .

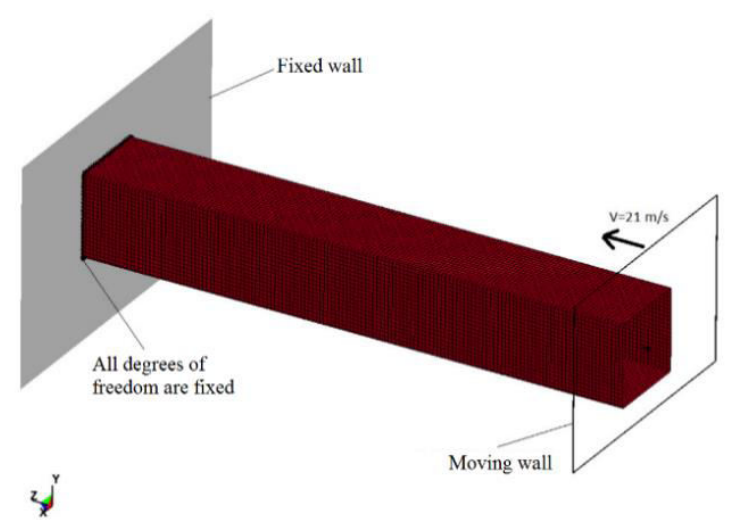

Figure 2. Finite element model of energy absorber

The automatic contact option was used to prevent the folding surfaces from penetrating into each other during deformation, and the friction coefficient was taken as 0.25 for dynamic and static conditions. In addition, the friction coefficient for the friction between the moving rigid plate and the tube was also taken as 0.25. Belytscho-Tsay shell elements with six degrees of freedom for each node and five integration points along the thickness was chosen for meshing the tube because of frequently used element type in the collision analysis for giving short solution times and approximate value assignments [9]. Consequently, the mesh size used in the analyses was chosen to be about twice the thickness value used for each energy absorber according to Ref.[10].

\section{Results and Discussion}

In this section, axial deformation behaviors of straight and truncated (with angles of $1,5^{\circ}$ and $3^{\circ}$ ) tube-like structures under dynamic loading conditions were investigated.For truncated and straight circular crosssection members, rigid wall force-displacement graph is given in Figure 3, the mean force-displacement and absorbed energy displacement graphs are given in Figure 4. The deformation patterns of straight and truncated (with angle of $1,5^{\circ}$ ) members with wall thicknesses of 4 and $6 \mathrm{~mm}$ are given in Figure 5.

It is seen from Figure 7 that the local maximum and minimum force values are comprised during the formation of the folds in the axial deformation of the energy absorbing members. In the formation of the first fold the rigid wall force value reaches its maximum and then decreases suddenly. During the formation of the second and subsequent folds, the force fluctuates between the local maximum and minimum values. 


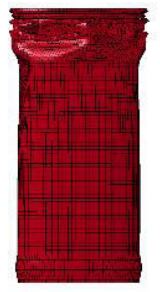

$0.2 \mathrm{~s}$

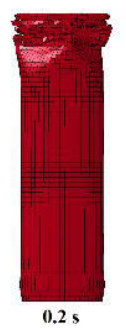

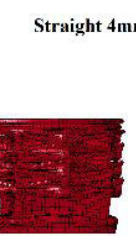

$0.4 \mathrm{~s}$

Straight $6 \mathrm{~mm}$

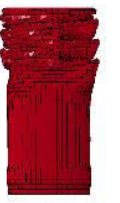

$0.4 \mathrm{~s}$

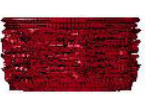

$0.6 \mathrm{~s}$

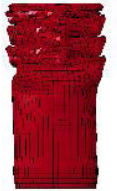

$0.6 \mathrm{~s}$

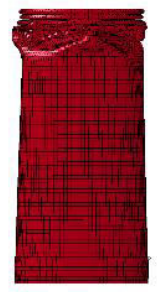

$0.2 \mathrm{~s}$

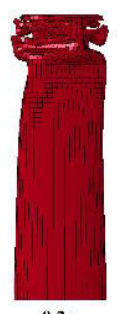

$0.2 \mathrm{~s}$

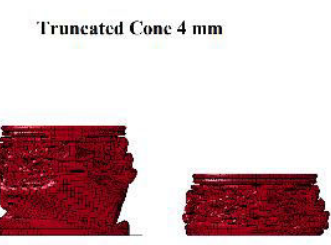

$0.6 \mathrm{~s}$

Truncated Cone 6 mm

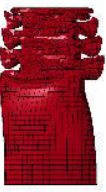

$0.4 \mathrm{~s}$

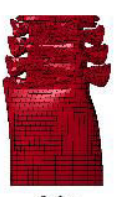

$0.6 \mathrm{~s}$

Figure 3. Deformation states of straight and truncated cone members versus time

Al Galib and Limam[17] emphasized this situation that, the initial force required to form the first fold of the member should be high because there is no deformation at the beginning of the tube. When the formation of the first fold, deformations occur in the member and that these deformations reduce the subsequent peak forces.

If the member has the capability to absorb the current impact energy, the force value reaches zero at the rigid wall force-displacement graph. Otherwise, the member loses its energy absorbing ability and starts to behave like a rigid body. When the rigid plate wall force-displacement graph is examined (Fig. 3), it is seen that the rigid wall force increases again towards the end of the analysis due to the loss of energy absorbing ability of members with $4 \mathrm{~mm}$ thickness.

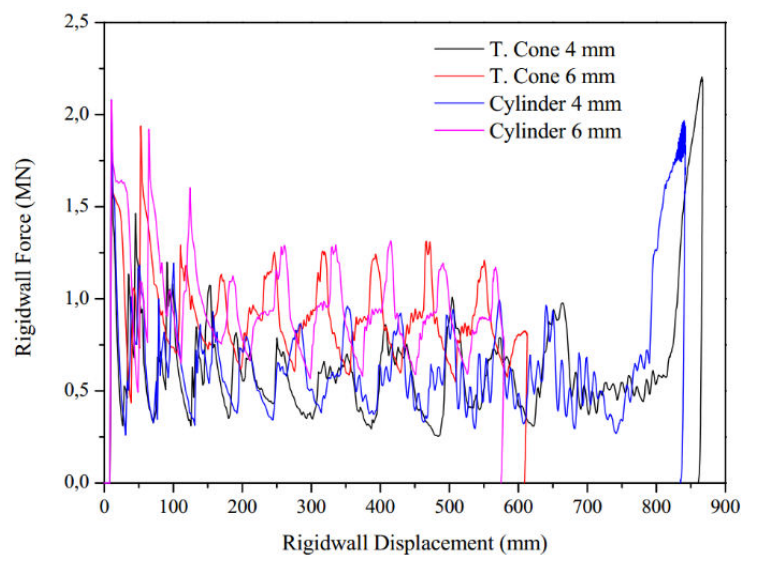

Figure 4. Rigid wall force-displacement fortruncatedand circular cross-section members 

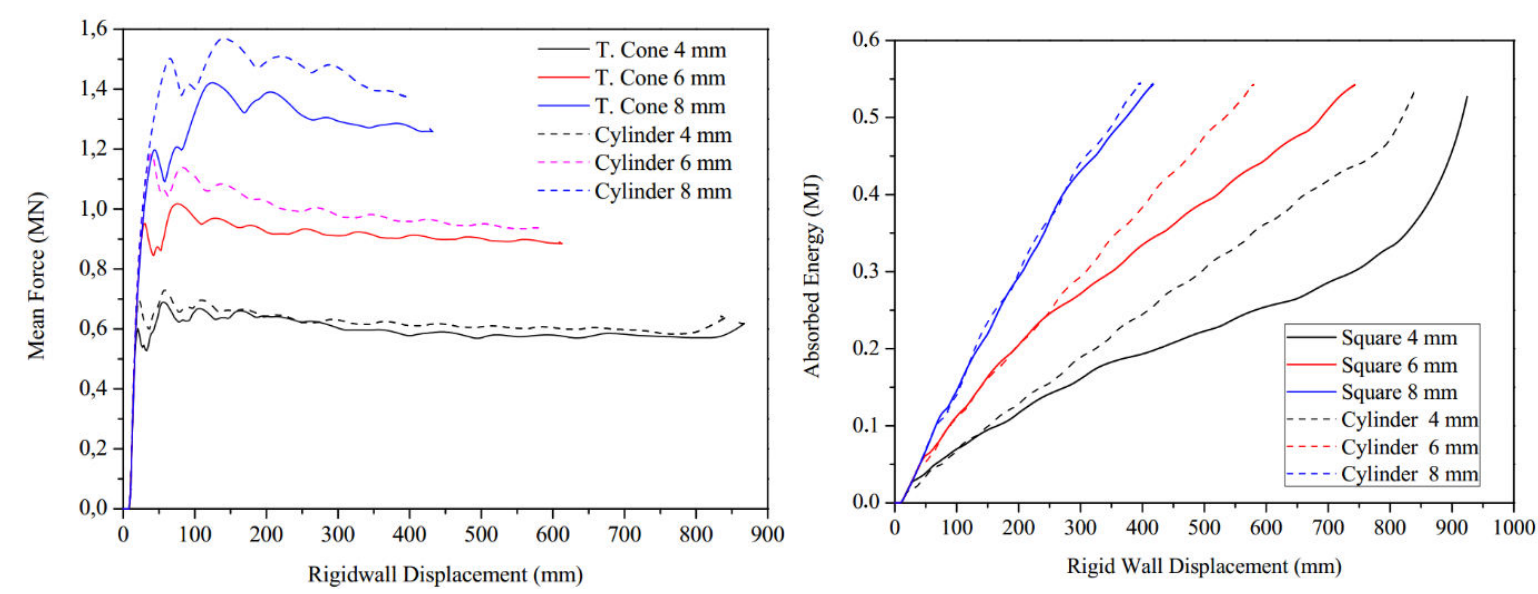

Figure 5. Mean force and absorbed energy versus displacement for $1.5^{\circ}$ tapered and straight members

According to the average deformation force-rigid plate displacement graph (Fig. 4), it is seen that the average deformation force is increases as the thickness increases because a rigid structure is obtained at the same member weight. Though the weight is the same, the member becomes more rigid as the thickness increases. In this study, axial deformation behaviors of equally weighted members were examined, hence the crosssectional dimensions change with increasing thickness. Even though the thickness and the cross-sectional properties of the members change, the amount of material required for plastic deformation remains the same. In this case, the increase of the average deformation force is due to the increase of the full plastic bending moment $M_{0}=\sigma_{0} h^{2} / 4$ required for buckling when the thickness increases. Figure 4 shows a comparison between straight and truncated members, it is seen that the average deformation force values of straight members are higher than those with truncated members with the same wall thicknesses.

The amount of maximum deformation and the change in initial peak force with respect to thickness and taper angle for the members in the form of straight and truncated cone are given in Figure 6.
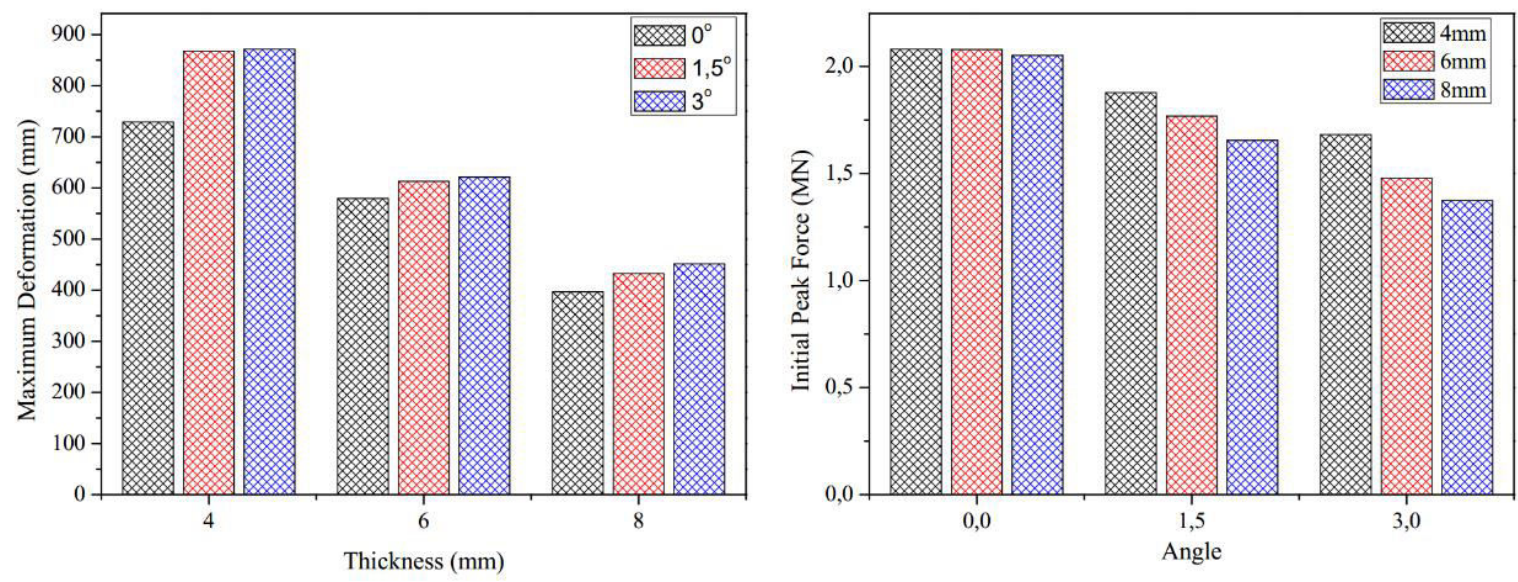

Figure 6. The effect of taper angle on maximum deformation of the member and initial peak force

As can be seen from the Figure 6, the amount of maximum deformation decreases as the thickness of the member increases. When the effect of the taper angle on the maximum deformation is examined for the same thickness elements, the amount of maximum deformation increases as the taper angle of the member increases.

The low initial peak force is desirable feature for energy absorbing members. Therefore, when the change of the first peak force with the taper angle is examined, it is seen that as the taper angle increases, the initial peak force decreases. The reduction in the initial peak force is very small for the straight elements, but this reduction is more pronounced for the truncated elements. The cross-sectional area of the member is decreased to provide the same weight as the thickness of the element increased. From the initial peak force vs angle graph, the initial peak force decreases even if the thickness of the member increases. 


\subsection{Optimization Procedure}

The effect levels of design parameters on total efficiency (TE) were determined according to experimental results with \%95 confidence level of analysis of variance (Table 1).Here, degree of freedom (DF), the sum of squares (SS), mean square (MS), F-ratio and the percent contribution ratio (PCR) is shown. The F-ratios and their PCR values have been considered to identify the significance levels of the design parameters. Table 1 shows that the most effective parameter is thickness of energy absorber with PCR of $60.52 \%$. The taper angle is secondary important with PCR of $29.01 \%$ on the total efficiency according to ANOVA results.

Table 1. ANOVA for $\mathrm{S} / \mathrm{N}$ ratios of total efficiency

\begin{tabular}{lccccc}
\hline Parameter & DF & SS & MS & F-ratio & \%PCR \\
\hline $\mathrm{T}$ & 2 & 8.109 & 4.0546 & 11.56 & 60.52 \\
$\mathrm{Ta}$ & 2 & 3.887 & 1.9434 & 5.54 & 29.01 \\
Error & 4 & 1.403 & 0.3507 & & 10.47 \\
Total & 8 & 13.399 & & & 100 \\
\hline
\end{tabular}

\subsection{Optimization of design parameters}

Design parameters for energy absorbers were optimized based on Taguchi method. Therefore, the-larger-thebetter approach was applied due to desire of maximum total efficiency (TE) which is determined as performance characteristic in finite element modelling. The design parameters giving optimum total efficiency value were determined in optimization study according to $\mathrm{S} / \mathrm{N}$ ratio [18]. In the Eq. (2), $n$ and $y$ shows the number of simulations and performance characteristic, respectively.

$$
S / N=-10 \log \left(1 / n \sum_{i=1}^{n} 1 / y_{i}^{2}\right)
$$

The total efficiency which is calculated with the data obtained from finite element analysis and $\mathrm{S} / \mathrm{N}$ ratios are given in Table 2. Statistical analyses and optimization studies were performed via Minitab software.

Table 2. FE simulation results and $\mathrm{S} / \mathrm{N}$ ratios

\begin{tabular}{ccccc}
\hline Exp. No & $\mathrm{T}$ & $\mathrm{Ta}$ & $\mathrm{TE}$ & $\mathrm{S} / \mathrm{N}$ \\
\hline 1 & 4 & 0 & 0.215605 & -13.3268 \\
2 & 4 & 1.5 & 0.233029 & -12.6518 \\
3 & 4 & 3 & 0.247227 & -12.1381 \\
4 & 6 & 0 & 0.267511 & -11.4532 \\
5 & 6 & 1.5 & 0.283052 & -10.9627 \\
6 & 6 & 3 & 0.366202 & -8.72558 \\
7 & 8 & 0 & 0.257682 & -11.7783 \\
8 & 8 & 1.5 & 0.257676 & -11.7785 \\
9 & 8 & 3 & 0.270111 & -11.0534 \\
\hline
\end{tabular}

$\mathrm{S} / \mathrm{N}$ ratios of parameters are considered in Taguchi optimization method. The highest $\mathrm{S} / \mathrm{N}$ ratio shows the optimum level of parameters according to "the-larger-the-better" approach. S/N ratios calculated by simulation results of total efficiency are shown in main effect plot (Fig. 7). Also, the variation of S/N ratios according to parameters is given in Table 3. It was shown that thickness is the most important thickness on TE when examining the main effect plots in Fig. 7 and the difference $(\Delta)$ between maximum and minimum values of $\mathrm{S} / \mathrm{N}$ ratios in Table 2. The optimum levels for minimum $\mathrm{TE}$ according to $\mathrm{S} / \mathrm{N}$ ratios were determined as $\mathrm{T} 2$, Ta3. 


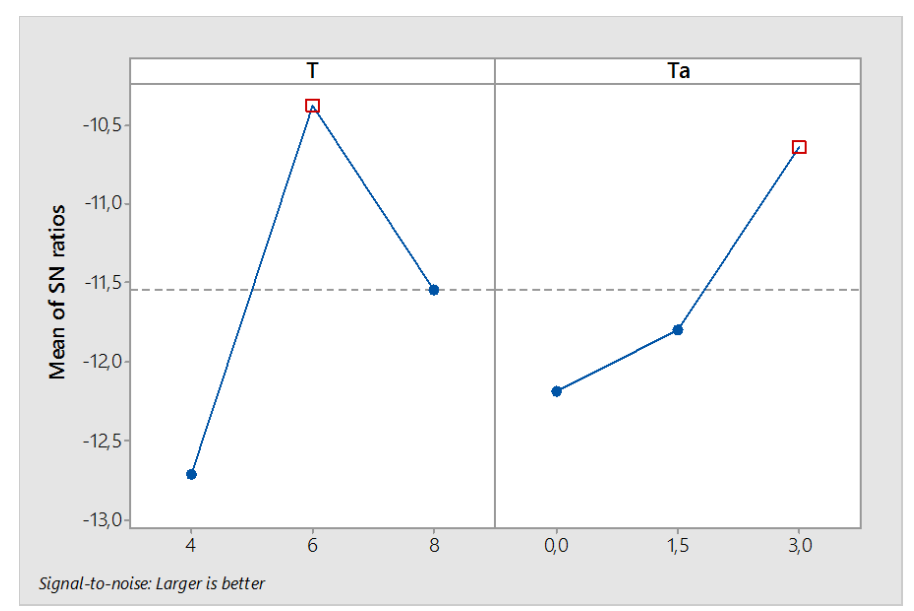

Figure 7. The main effect plot for SN ratio of total efficiency

Table 3. Response table of $\mathrm{S} / \mathrm{N}$ ratios for total efficiency

\begin{tabular}{llllc}
\hline \multirow{2}{*}{ Parameter } & \multicolumn{4}{c}{ S/N ratios } \\
\cline { 2 - 5 } & Level 1 & Level 2 & Level 3 & $\Delta$ \\
\hline Thickness & -12.71 & $-10.38^{*}$ & -11.54 & 2.33 \\
Taper angle & -12.19 & -11.8 & $-10.64 *$ & 1.55 \\
\hline *Optimum levels of parameters \\
\hline
\end{tabular}

Average of FE simulation results performed at optimal levels are evaluated by Eq. (3) to forecast the mean for the improvement conditions. Eq. (1) which is the expression of calculated total efficiency (TE) is derived from Eq. (4).

$$
\begin{aligned}
& \eta_{G}=\bar{\eta}_{G}+\left(\bar{T}_{0}-\bar{\eta}_{G}\right)+\left(T a_{0}-\bar{\eta}_{G}\right) \\
& T E_{c a l}=10^{-\eta_{G} / 20}
\end{aligned}
$$

Where, $\eta_{G}$ is the $\mathrm{S} / \mathrm{N}$ ratio calculated at optimal level of factors $(\mathrm{dB}), \bar{\eta}_{G}$ is the mean $\mathrm{S} / \mathrm{N}$ ratio of all parameters (dB), $\overline{A_{0}}$ and $\overline{B_{0}}$ are the mean $\mathrm{S} / \mathrm{N}$ ratio once thickness and taper angle are at optimum levels, and $T E_{c a l}$ is the calculated value. Consequently, $\eta_{G}$ and $T E_{c a l}$ for optimum design parameters were determined as $-9.4785 \mathrm{~dB}$ and 0.3358, respectively. Lastly, confirmation experiments with FE modelling were done by using the optimum design parameters after the determination of these factors for $T E$ and thus reliability of the optimization has been confirmed. The FE simulations conducted by considering the confidence interval $(C I)$ calculated from Eqn. (5) and (6) [19].

$$
\begin{aligned}
& C I=\sqrt{F_{\alpha,(1, V e)} V_{e}\left(1 / \eta_{e f f}+1 / r\right)} \\
& \eta_{\text {eff }}=N /\left(1+v_{T}\right)
\end{aligned}
$$

Where; $\boldsymbol{F}_{\boldsymbol{\alpha},(1, \boldsymbol{V}) \text { is }}$ is the F-ratio at the $95 \%$ significance level, $\alpha$ is the importance level, $v_{e}$ is the degree of freedom of the error, $V e$ is the error variance, $n_{\text {eff }}$ is the effective number of replications, $r$ is the number of replications for the verification test. In Equation 6, $\mathrm{N}$ is the total number of experiments and $v_{T}$ is the total main factor of the degree of freedom. Total efficiency obtained with FE simulation $\left(T E_{\text {exp }}\right)$, calculated total efficiency $\left(T E_{c a l}\right)$, and $\mathrm{S} / \mathrm{N}$ ratios $\left(\eta_{\text {exp }}, \eta_{c a l}\right)$ for $T E$ are given by comparing between simulation results and calculated values (Table 4). Table 4 shows differences between confirmation experiment results and calculated values and their S/N ratios obtained by using Eqn. 2 and 3. As can be seen from the Table4, a difference of $0.7529 \mathrm{~dB}$ is under the confidence interval of $1.6893 \mathrm{~dB}$ for total efficiency. Thus, the optimum levels of design parameters for total efficiency were approved as confident.

Table 4. Comparison of simulation results and calculated values

\begin{tabular}{|l|l|l|l|l|l|}
\hline \multicolumn{2}{|l|}{ Simulation results } & \multicolumn{2}{l|}{ Calculated value } & \multicolumn{2}{l|}{ Difference } \\
\hline$T E_{\text {exp }}(\mu \mathrm{m})$ & $\eta_{\text {exp }}(\mathrm{dB})$ & $T E_{\text {cal }}(\mu \mathrm{m})$ & $\eta_{\text {cal }}(\mathrm{dB})$ & $T E_{\text {exp }}-T E_{\text {cal }}$ & $\eta_{\text {exp }}-\eta_{\text {cal }}$ \\
\hline 0.3662 & -8.7256 & 0.3358 & -9.4785 & 0.0304 & 0.7529 \\
\hline
\end{tabular}




\section{Conclusions}

In this study, the axial deformation behaviors of the energy absorbing members in the form of straight and truncated (with angles of $1,5^{\circ}$ and $3^{\circ}$ ) tubes were investigated under the impact force acting on the existing primary energy absorbing member.

Initially, deformation performances were compared with the condition that the weight and length of the energy absorbing members were the same. As a result of comparison, it was found that the average deformation force values of straight members are higher than those with truncated members with the same wall thicknesses. When the thickness of the member is increased, the average deformation force increased due to the increase of the full plastic bending moment required for buckling. Though the weight is the same, the member becomes more rigid as the thickness increases.

It was realized that the amount of maximum deformation decreases as the thickness of the member increases and increases as the taper angle of the member increases for the same thickness elements. The low initial peak force is desirable feature for energy absorbing members. Therefore, when the change of the first peak force with the taper angle is examined, it is seen that as the taper angle increases, the initial peak force decreases.

Consequently, design parameters for energy absorbers were optimized based on Taguchi method. Therefore, the-larger-the-better approach was applied due to desire of maximum total efficiency (TE) which is determined as performance characteristic of the member. The design parameters giving optimum total efficiency value were determined in optimization study according to $\mathrm{S} / \mathrm{N}$ ratio. According to optimization study, the optimum energy absorbing member was determined as a truncated member with the $6 \mathrm{~mm}$ thickness and taper angle of $1.5^{\circ}$. The effects of taper angle and thickness on total crush efficiency of the member calculated as $10.38 \%$ and $10.64 \%$ respectively.

\section{References}

[1] E. Martinez, DavidTyrell, R. Rancatore, R. Stringfellow, and G. Amar, "A crush zone design for an existing passenger rail cab car," in Proceedings of 2005 ASME International Mechanical Engineering Congress \& Exposition, 2004, pp. 85-94.

[2] K. Jacobsen, D. Tyrell, and B. Perlman, "Impact Tests of Crash Energy Management Passenger Rail Cars: Analysis and Structural Measurements," in ASME 2004 International Mechanical Engineering Congress and Exposition, 2004, pp. 97-105.

[3] W. Abramowicz and N. Jones, "Dynamic progressive buckling of circular and square tubes," Int. J. Impact Eng., vol. 4, no. 4, pp. 243-270, 1986.

[4] S. Simunovic, J. Shaw, and G. a Aramayo, "Material Modeling Effects on Impact Deformation of Ultralight Steel Auto Body," SAE Tech. Pap., no. 724, 2000.

[5] A. A. Nia and J. H. Hamedani, "Comparative analysis of energy absorption and deformations of thin walled tubes with various section geometries," Thin-Walled Struct., vol. 48, no. 12, pp. 946-954, 2010.

[6] M. Langseth, O. S. Hopperstad, and T. Berstad, "Crashworthiness of aluminium extrusions: validation of numerical simulation, effect of mass ratio and impact velocity," Int. J. Impact Eng., vol. 22, no. 910, pp. 829-854, 1999.

[7] A. Tasdemirci, "The effect of tube end constraining on the axial crushing behavior of an aluminum tube," Mater. Des., vol. 29, no. 10, pp. 1992-2001, 2008.

[8] R. Gümrük and S. Karadeniz, "The influences of the residual forming data on the quasi-static axial crash response of a top-hat section,” Int. J. Mech. Sci., vol. 51, no. 5, pp. 350-362, 2009.

[9] R. Gümrük and S. Karadeniz, "A numerical study of the influence of bump type triggers on the axial crushing of top hat thin-walled sections," Thin-Walled Struct., vol. 46, no. 10, pp. 1094-1106, 2008.

[10] G. Chen, X. M. Chen, and M. F. Shi, "Experimental and Numerical Studies of Crash Trigger Sensitivity in Frontal Impact," SAE Tech. Pap., no. 724, 2005.

[11] A. G. Hanssen, M. Langseth, and O. S. Hopperstad, "Static and dynamic crushing of circular aluminium extrusions with aluminium foam filler," Int. J. Impact Eng., vol. 24, no. 5, pp. 475-507, 2000 . 
[12] G. Mayville, R. A., Rancatore, R. J., Stringfellow, R. G., Amar, "Repair of Budd Pioneer Coach Car Crush Zones," Cambridge, MA, 2007.

[13] D. A. Skobir, "High-strength low-alloy (HSLA) steels," Mater. Technol., vol. 45, no. 4, pp. 295-301, 2011.

[14] J. Patel, C. Klinkenberg, and K. Hulka, "Hot rolled HSLA strip steels for automotive and construction applications," Niobium Sci. Technol., no. Grade 100, pp. 647-674, 2001.

[15] R. Mayville, A. D. Little, K. Johnson, and P. Engineering, "The Development of a Rail Passenger Coach Car Crush Zone," in Proceedings of the 2003 IEEE/ASME Joint Rail Conference, 2003, pp. 18.

[16] J. O. Hallquist, LS-DYNA users manual. Livermore, California: Livemore Software Technology Corporation, 1998.

[17] D. Al Galib and A. Limam, "Experimental and numerical investigation of static and dynamic axial crushing of circular aluminum tubes," Thin-Walled Struct., vol. 42, no. 8, pp. 1103-1137, 2004.

[18] G. Taguchi, S. Chowdhury, Y. Wu, Taguchi's Quality Engineering Handbook, John Wiley \& Sons, Inc., New Jersey, USA, 2005.

[19] M. Günay, M.E. Korkmaz, Optimization of Honing Parameters for Renewal of Cylinder Liners. GU J Sci 30(1), 111-119, 2017. 